\title{
SUPER NESTED ARRAYS: SPARSE ARRAYS WITH LESS MUTUAL COUPLING THAN NESTED ARRAYS
}

\author{
Chun-Lin Liu ${ }^{1}$ and P. P. Vaidyanathan ${ }^{2}$ \\ Dept. of Electrical Engineering, 136-93 \\ California Institute of Technology, Pasadena, CA 91125, USA \\ E-mail: cl.liu@ caltech.edu ${ }^{1}$ and ppvnath@systems.caltech.edu ${ }^{2}$
}

\begin{abstract}
In array processing, mutual coupling between sensors has an adverse effect on the estimation of parameters (e.g., DOA). Sparse arrays, such as nested arrays, coprime arrays, and minimum redundancy arrays (MRAs), have reduced mutual coupling compared to uniform linear arrays (ULAs). With $N$ denoting the number of sensors, these sparse arrays offer $O\left(N^{2}\right)$ freedoms for source estimation because their difference coarrays have $O\left(N^{2}\right)$-long ULA segments. These arrays have different shortcomings: coprime arrays have holes in the coarray, MRAs have no closed-form expressions, and nested arrays have relatively large mutual coupling. This paper introduces a new array called the super nested array, which has all the good properties of the nested array, and at the same time reduces mutual coupling significantly. For fixed $N$, the super nested array has the same physical aperture, and the same hole-free coarray as does the nested array. But the number of sensor pairs with separation $\lambda / 2$ is significantly reduced. Many theoretical properties are proved and simulations are included to demonstrate the superior performance of these arrays.
\end{abstract}

Index Terms - Nested arrays, coprime arrays, super nested arrays, mutual coupling, DOA estimation.

\section{INTRODUCTION}

Array processing plays a significant role in many applications such as radar [1], astronomy [2], tomography [2], and communications [3]. Sensor measurements enable us to extract source profiles, such as direction-of-arrival (DOA), radial velocity, range, power, and polarization [1-4]. However, in practice, electromagnetic characteristics cause mutual coupling between sensors, making the sensor responses interfere with each other [1]. This has an adverse effect on the estimation of parameters (e.g., DOA). State-of-the-art approaches aim to decouple (or "remove") the effect of mutual coupling from the received data by using proper mutual coupling models [5-12]. Such methods are usually computationally expensive, and sensitive to model mismatch.

An altogether different approach to reduce the effect of mutual coupling is to use sparse arrays, in which the number of sensor pairs with small separations (small multiples of $\lambda / 2$ ) is much fewer than in uniform linear arrays (ULAs). This paper is based on this theme. Sparse arrays such as nested arrays [13], coprime arrays [14], minimum redundancy arrays (MRA) [15] and minimum hole arrays (MHAs) [16] have reduced mutual coupling compared to ULAs. They also offer another important advantage over ULAs: with $N$ denoting the number of sensors, these sparse arrays offer $O\left(N^{2}\right)$ free-

This work was supported in parts by the ONR grant N00014-15-1-2118, and the California Institute of Technology. doms for source estimation because their difference coarrays have $O\left(N^{2}\right)$-long ULA segments $[13,14]$. That is, the number of uncorrelated source directions that can be estimated is increased from $N-1$ to $O\left(N^{2}\right)$. Typically a MUSIC algorithm is performed in the difference coarray domain to achieve this $[13,14]$.

In practice, these well-known sparse arrays have some shortcomings: MRAs and MHAs do not have simple closed-form expressions for the array geometry, and the sensor locations are usually found from tabulated entries [15-17]. Coprime arrays have holes in the coarray, so that the ULA part of the coarray is smaller than those of the nested array and the MRA [14]. Finally nested arrays, by definition, contain a dense ULA in the physical array, resulting in significantly higher mutual coupling than coprime arrays and MRAs [13]. We will not elaborate further on MRA because there are no closedform expressions for the sensor locations.

The main aim of this paper is to introduce a new array configuration called the super nested array, which has all the good properties of the nested array, and at the same time achieves reduced mutual coupling by redistributing the elements of the dense ULA part of the parent nested array. We will show how to systematically determine the appropriate sensor locations for any $N$. For fixed $N$ (number of sensors) the super nested array has the same physical aperture as the parent nested array. Furthermore, its difference coarray is exactly identical to that of the nested array and is, in particular, free from holes. This allows us to perform coarray MUSIC using the entire coarray as in [13]. However, unlike the nested array, the number of sensor pairs with separation $\lambda / 2$ is significantly reduced. More quantitative statements of these properties will be given in this paper based on the weight function $w(m)$ of the sparse array (where $w(m)$ is the number of pairs of elements with element-spacing $m \lambda / 2$ ). Several other properties of the new array geometry will also be established.

Section 2 reviews sparse array processing and mutual coupling models. Super nested arrays are then introduced in Section 3. In Section 4, we prove that the coarray of the super nested array is identical to that of the parent nested array (hence hole-free). We also show that the weight function $w(1)$ of the super nested array is much smaller than that of the nested array. The improved performance of super nested arrays under mutual coupling will be demonstrated through examples in Section 5.

\section{PRELIMINARIES}

\subsection{Sparse Array Processing}

Assume $D$ monochromatic far-field sources impinge on the sensor array, where the sensor locations are $n d$. Here $n$ belongs to some integer set $\mathbb{S}$ and $d=\lambda / 2$ denotes the minimum distance between 
(a)

Dense ULA, $N_{1}$ sensors

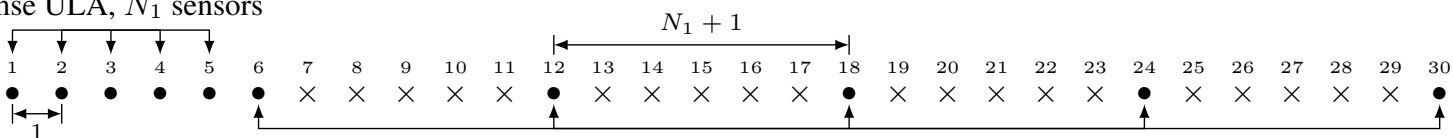

Sparse ULA, $N_{2}$ sensors

(b)

$N$ sensors with inter-element spacing $M$

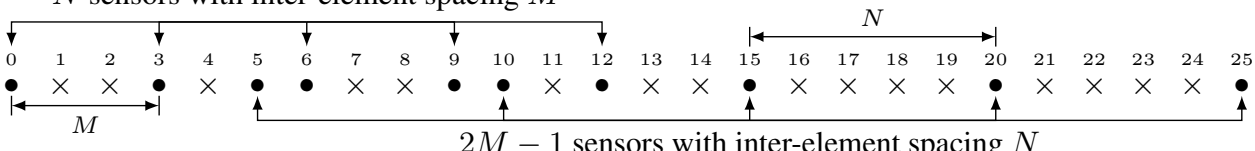

Fig. 1. (a) A nested array with $N_{1}=N_{2}=5$, (b) a coprime array with $M=3$ and $N=5$, and (c) a super nested array with $N_{1}=N_{2}=5$. Bullets stand for physical sensors and crosses represent empty space.

sensors. For the $i$ th source, its complex amplitude is written as $A_{i}$ and its direction-of-arrival (DOA) is denoted by $\theta_{i} \in[-\pi / 2, \pi / 2)$. The measurement vector $\mathbf{x}_{\mathbb{S}}$ on the sensor array $\mathbb{S}$ can be modeled as follows:

$$
\mathbf{x}_{\mathbb{S}}=\sum_{i=1}^{D} A_{i} \mathbf{v}_{\mathbb{S}}\left(\bar{\theta}_{i}\right)+\mathbf{n}_{\mathbb{S}},
$$

where $\mathbf{v}_{\mathbb{S}}\left(\bar{\theta}_{i}\right)=\left[e^{j 2 \pi \bar{\theta}_{i} n}\right]_{n \in \mathbb{S}}$ are steering vectors and $\mathbf{n}_{\mathbb{S}}$ is the additive noise term. $\bar{\theta}_{i}=(d / \lambda) \sin \theta_{i}$ is the normalized DOA, with $-1 / 2 \leq \bar{\theta}_{i}<1 / 2$. The parameters $A_{i}$ and $\mathbf{n}_{\mathbb{S}}$ are assumed to be zero-mean, uncorrelated random variables with $\mathbb{E}\left[A_{i} A_{j}^{*}\right]=\sigma_{i}^{2} \delta_{i, j}$ and $\mathbb{E}\left[\mathbf{n}_{\mathbb{S}} \mathbf{n}_{\mathbb{S}}^{H}\right]=\sigma^{2} \mathbf{I}$. $\bar{\theta}_{i}$ is considered to be fixed but unknown.

The covariance matrix of $\mathbf{x}_{\mathbb{S}}$ can be expressed as

$$
\mathbf{R}_{\mathbf{x}_{\mathbb{S}}}=\sum_{i=1}^{D} \sigma_{i}^{2} \mathbf{v}_{\mathbb{S}}\left(\bar{\theta}_{i}\right) \mathbf{v}_{\mathbb{S}}^{H}\left(\bar{\theta}_{i}\right)+\sigma^{2} \mathbf{I} .
$$

Since the entries in $\mathbf{v}_{\mathbb{S}}\left(\bar{\theta}_{i}\right) \mathbf{v}_{\mathbb{S}}^{H}\left(\bar{\theta}_{i}\right)$ are of the form $e^{j 2 \pi \bar{\theta}_{i}\left(n_{1}-n_{2}\right)}$, where $n_{1}, n_{2} \in \mathbb{S}$, it enables us to reshape (2) into an autocorrelation vector $\mathbf{x}_{\mathbb{D}}$ as in $[13,18]$

$$
\mathbf{x}_{\mathbb{D}}=\sum_{i=1}^{D} \sigma_{i}^{2} \mathbf{v}_{\mathbb{D}}\left(\bar{\theta}_{i}\right)+\sigma^{2} \mathbf{e}_{0},
$$

where $\left\langle\mathbf{e}_{0}\right\rangle_{m}=\delta_{m, 0}$ for $m \in \mathbb{D}$ and $\delta_{i, j}$ is the Kronecker delta. Here $\langle\cdot\rangle_{m}$ denotes the signal value on the support $m \in \mathbb{D}$ [19]. For instance, if $\mathbb{D}=\{-1,0,1\}$ and $\mathbf{x}_{\mathbb{D}}=[1-j, 2,1+j]^{T}$, then $\left\langle\mathbf{x}_{\mathbb{D}}\right\rangle_{-1}=1-j,\left\langle\mathbf{x}_{\mathbb{D}}\right\rangle_{0}=2$, and $\left\langle\mathbf{x}_{\mathbb{D}}\right\rangle_{1}=1+j . \mathbb{D}$ is called the difference coarray of $\mathbb{S}$, defined by

Definition 1 (Difference coarray). For a sparse array specified by an integer set $\mathbb{S}$, its difference coarray $\mathbb{D}$ is defined as $\mathbb{D}=\left\{n_{1}-n_{2} \mid n_{1}, n_{2} \in \mathbb{S}\right\}$.

In other words, the original model (1) in the physical array domain $\mathbb{S}$, is converted into another model (3) in the difference coarray domain $\mathbb{D}$. An array is said to be a restricted array [15] if the difference coarray is hole free. The following definitions are useful:

Definition 2 (Uniform DOF). Given an array $\mathbb{S}$, let $\mathbb{U}$ denote the maximum central ULA segment of its difference coarray. The number of elements in $\mathbb{U}$ is called the uniform degrees of freedom or "uniform DOF” of $\mathbb{S}$.
If the uniform DOF is $\mathcal{F}$, then the number of uncorrelated sources that can be identified by using coarray MUSIC is $(\mathcal{F}-1) / 2$ $[13,18]$.

Definition 3 (Weight functions). The weight function $w(m)$ of an array $\mathbb{S}$ is defined as the number of sensor pairs that lead to coarray index m. Namely,

$$
w(m)=\left|\left\{\left(n_{1}, n_{2}\right) \in \mathbb{S}^{2} \mid n_{1}-n_{2}=m\right\}\right|, \quad m \in \mathbb{D} .
$$

Nested arrays [13] and coprime arrays [14] are two useful types of sparse arrays which provide $O\left(N^{2}\right)$ uniform DOF using $N$ sensors [13,14, 18, 20-22], and are demonstrated in Figs. 1(a), (b). Of these, only nested arrays have hole-free coarrays. Both arrays have simple expressions for sensor locations. For nested arrays, the sensor locations are given by

$$
\mathbb{S}_{\text {nested }}=\left\{1, \ldots, N_{1},\left(N_{1}+1\right), \ldots N_{2}\left(N_{1}+1\right)\right\},
$$

where $N_{1}$ and $N_{2}$ are positive integers. The sensor locations for coprime arrays are

$$
\mathbb{S}_{\text {coprime }}=\{0, M, \ldots,(N-1) M, N, \ldots,(2 M-1) N\},
$$

where $M$ and $N$ are a coprime pair of positive integers.

\subsection{Mutual Coupling}

Equation (1) assumes that the sensors do not interfere with each other. In practice, any sensor output is influenced by its neighboring elements, due to mutual electromagnetic coupling.

Mutual coupling can be incorporated into (1) as follows:

$$
\mathbf{x}_{\mathbb{S}}=\sum_{i=1}^{D} A_{i} \mathbf{C} \mathbf{v}_{\mathbb{S}}\left(\bar{\theta}_{i}\right)+\mathbf{n}_{\mathbb{S}},
$$

where $\mathbf{C}$ is a mutual coupling matrix that can be obtained from electromagnetics. The mutual coupling matrix $\mathbf{C}$ can be approximated by a $B$-banded symmetric Toeplitz matrix in the ULA configuration $[5,7]$ as follows:

$$
\langle\mathbf{C}\rangle_{n_{1}, n_{2}}= \begin{cases}c_{\left|n_{1}-n_{2}\right|}, & \text { if }\left|n_{1}-n_{2}\right| \leq B, \\ 0, & \text { otherwise, }\end{cases}
$$

where $n_{1}, n_{2} \in \mathbb{S}$ and coupling coefficients $c_{0}, c_{1}, \ldots, c_{B}$ satisfy $1=c_{0}>\left|c_{1}\right|>\left|c_{2}\right|>\cdots>\left|c_{B}\right|$. It is assumed that the magnitudes of coupling coefficients are inversely proportional to their 
sensor separations [5], i.e. $\left|c_{k} / c_{\ell}\right|=\ell / k$. There also exist more sophisticated models based on electromagnetics [8,23].

To counteract mutual coupling, the standard approach is to estimate mutual coupling and source profiles based on the received data and particular mutual coupling models $[5,7-10,12]$. For instance, BouDaher et al. considered DOA estimation with coprime arrays in the presence of mutual coupling [12]. Their algorithm jointly estimated the mutual coupling matrix $\mathbf{C}$, the source power, and the DOA under certain optimization criterion. At the expense of some extra computations, this approach estimates the true DOA satisfactorily. In principle, all of the above decoupling methods are applicable with the super nested arrays to be developed in this paper, and can only improve the performance further.

\section{SENSOR LOCATIONS IN SUPER NESTED ARRAYS}

In this section, we develop super nested arrays. For fixed number of sensors, these have the same physical aperture and the same difference coarray enjoyed by nested arrays (in particular there are no holes in coarray). But they have reduced mutual coupling because of smaller values of the crucial weight function $w(1)$.

To develop some feeling for super nested arrays, let us consider the super nested array with $N_{1}=N_{2}=5$, as shown in Fig. 1(c). It can be observed that there are $N_{1}+N_{2}=10$ sensors and the total aperture is 29 . The difference coarray for (c) comprises consecutive integers from -29 to 29 (Section 4). Note that these array profiles are identical to those for the nested array in Fig. 1(a). The only difference is that, super nested arrays are qualitatively sparser than nested arrays and mutual coupling effects are reduced as we shall see. Here is the formal definition:

Definition 4 (Super nested arrays). Assume $N_{1}$ and $N_{2}$ are integers satisfying $N_{1} \geq 4$ and $N_{2} \geq 3$. Super nested arrays are specified by the integer set $\mathbb{S}^{(2)}$, defined by

$$
\mathbb{S}^{(2)}=\mathbb{X}_{1}^{(2)} \cup \mathbb{Y}_{1}^{(2)} \cup \mathbb{X}_{2}^{(2)} \cup \mathbb{Y}_{2}^{(2)} \cup \mathbb{Z}_{1}^{(2)} \cup \mathbb{Z}_{2}^{(2)},
$$

where

$$
\begin{aligned}
& \mathbb{X}_{1}^{(2)}=\left\{1+2 \ell \mid 0 \leq \ell \leq A_{1}\right\}, \\
& \mathbb{Y}_{1}^{(2)}=\left\{\left(N_{1}+1\right)-(1+2 \ell) \mid 0 \leq \ell \leq B_{1}\right\}, \\
& \mathbb{X}_{2}^{(2)}=\left\{\left(N_{1}+1\right)+(2+2 \ell) \mid 0 \leq \ell \leq A_{2}\right\}, \\
& \mathbb{Y}_{2}^{(2)}=\left\{2\left(N_{1}+1\right)-(2+2 \ell) \mid 0 \leq \ell \leq B_{2}\right\}, \\
& \mathbb{Z}_{1}^{(2)}=\left\{\ell\left(N_{1}+1\right) \mid 2 \leq \ell \leq N_{2}\right\}, \\
& \mathbb{Z}_{2}^{(2)}=\left\{N_{2}\left(N_{1}+1\right)-1\right\} .
\end{aligned}
$$

The parameters $A_{1}, B_{1}, A_{2}$, and $B_{2}$ are defined as

$$
\left(A_{1}, B_{1}, A_{2}, B_{2}\right)= \begin{cases}(r, r-1, r-1, r-2), & \text { if } N_{1}=4 r, \\ (r, r-1, r-1, r-1), & \text { if } N_{1}=4 r+1, \\ (r+1, r-1, r, r-2), & \text { if } N_{1}=4 r+2, \\ (r, r, r, r-1), & \text { if } N_{1}=4 r+3,\end{cases}
$$

where $r$ is an integer.

First we will verify that Definition 4 leads to Fig. 1(c) exactly. Setting $N_{1}=N_{2}=5$ in Definition 4 yields $A_{1}=1, B_{1}=A_{2}=$ $B_{2}=0$, and

$$
\begin{gathered}
\mathbb{X}_{1}^{(2)}=\{1,3\}, \mathbb{Y}_{1}^{(2)}=\{5\}, \mathbb{X}_{2}^{(2)}=\{8\}, \mathbb{Y}_{2}^{(2)}=\{10\} \\
\mathbb{Z}_{1}^{(2)}=\{12,18,24,30\}, \mathbb{Z}_{2}^{(2)}=\{29\}
\end{gathered}
$$

which are the sensors in Fig. 1(c). Notice that Definition 4 can be used to find sensor locations for arbitrarily large $N_{1}$ and $N_{2}$. In addition, it can be inferred from Definition 4 that $\mathbb{X}_{1}^{(2)}, \mathbb{Y}_{1}^{(2)}, \mathbb{X}_{2}^{(2)}$, $\mathbb{Y}_{2}^{(2)}$, and $\mathbb{Z}_{1}^{(2)}$ are all ULAs with inter-element spacing 2, 2, 2, 2, and $N_{1}+1$, respectively.

Next, the relationship between nested arrays and super nested arrays is elaborated using Fig. 1. Most sensor locations remain unchanged, except for some sensor locations. In this example, we start with the parent nested array in Fig. 1(a) and then relocate some sensors, from location 2 to 8,4 to 10 , and 6 to 29 , yielding the super nested array in Fig. 1(c).

\section{COARRAY PROPERTIES}

The importance of super nested arrays arises from the three properties given below in Theorem 1, Corollary 1, and Theorem 2 .

Theorem 1. Super nested arrays are restricted arrays, i.e., they have hole-free difference coarrays.

Proof. The proof is quite involved and can be found in [24].

Corollary 1. Super nested arrays have the same coarray as their parent nested arrays.

Proof. According Definition 4, super nested arrays share the same boundary points, located at 1 and $N_{2}\left(N_{1}+1\right)$, as their parent nested arrays. In addition, both of them are restricted arrays (Theorem 1). Therefore, they possess the same coarray.

Theorem 2. Let $\mathbb{S}^{(2)}$ be a super nested array with $N_{1} \geq 4, N_{2} \geq 3$. Its weight function $w(m)$ satisfies

$w(1)=\left\{\begin{array}{ll}2, & \text { if } N_{1} \text { is even, } \\ 1, & \text { if } N_{1} \text { is odd. }\end{array}, \quad w(2)= \begin{cases}N_{1}-3, & \text { if } N_{1} \text { is even } \\ N_{1}-1, & \text { if } N_{1} \text { is odd }\end{cases}\right.$

$w(3)= \begin{cases}3, & \text { if } N_{1}=4,6 \\ 4, & \text { if } N_{1} \text { is even }, N_{1} \geq 8, \\ 1, & \text { if } N_{1} \text { is odd },\end{cases}$

For comparison, the first three weight function for nested arrays $[13]$ and coprime arrays $[14,18]$ are

$$
\begin{aligned}
& \text { Nested: } \quad w(1)=N_{1}, \quad w(2)=N_{1}-1, \quad w(3)=N_{1}-2, \\
& \text { Coprime: } \quad w(1)=2, \quad w(2)=2, \quad w(3)=2,
\end{aligned}
$$

where $N_{1}, N_{2}$ for nested arrays and $M, N$ for coprime arrays are sufficiently large. Clearly, super nested arrays have much smaller $w(1)$ and $w(3)$ compared to nested arrays. Although coprime arrays have smaller weights, they have holes in the coarray. Theorem 2 indicates that super nested arrays are qualitatively sparser than nested arrays and comparable to coprime arrays, so that mutual coupling is much less severe. The proof of the expression for $w(1)$ in Theorem 2 is given below. The proof for $w(2)$ and $w(3)$ can be found in [24].

Prooffor $w(1)$. According to Definition $4, \mathbb{X}_{1}^{(2)}, \mathbb{Y}_{1}^{(2)}, \mathbb{X}_{2}^{(2)}, \mathbb{Y}_{2}^{(2)}$, and $\mathbb{Z}_{1}^{(2)}$ are all ULA with sensor separation at least 2. Besides, their array apertures do not overlap. It is sufficient to consider their boundary points. Whenever $N_{1}$ is even or odd, the sensors at $N_{2}\left(N_{1}+1\right)-1$ and $N_{2}\left(N_{1}+1\right)$ contribute to one pair in $w(1)$. 


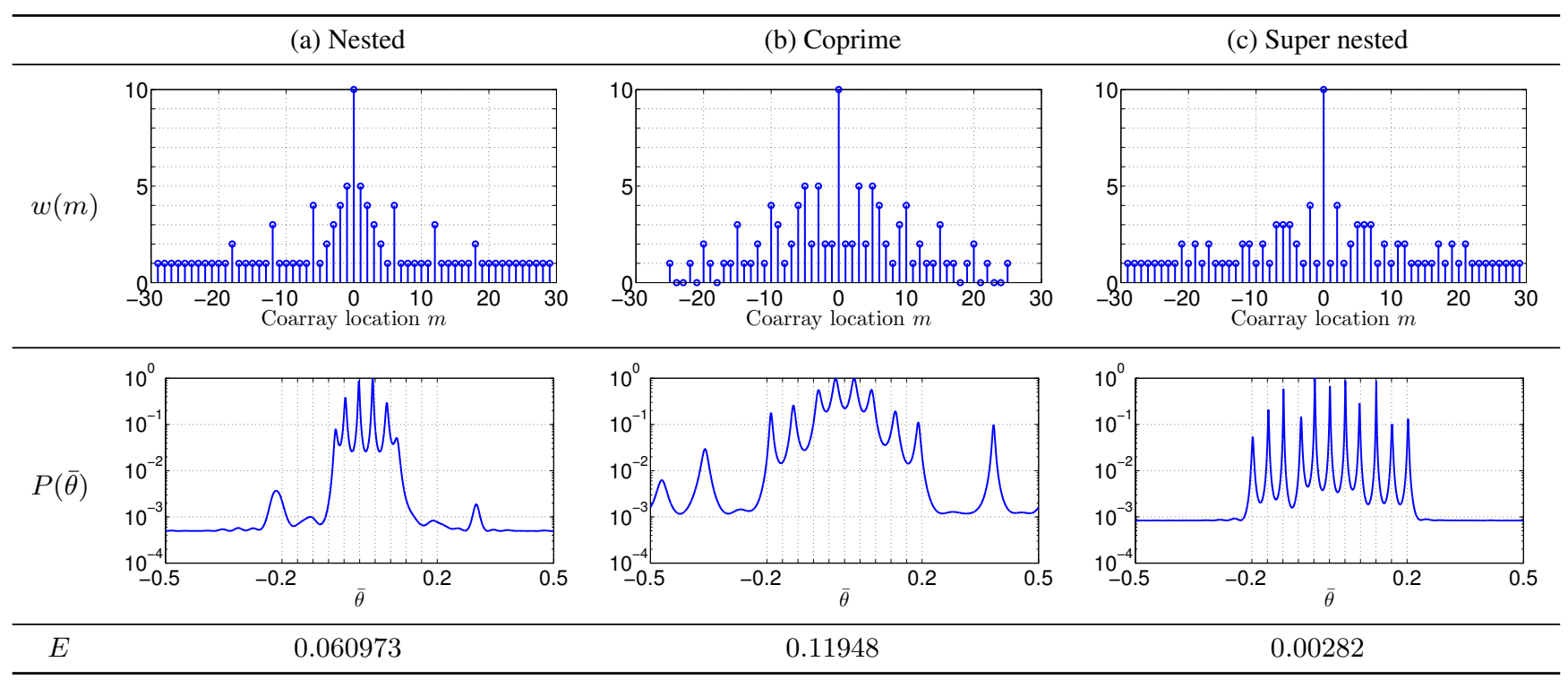

Fig. 2. Comparison among (a) nested arrays, (b) coprime arrays, and (c) super nested arrays in the presence of mutual coupling. The MUSIC spectra $P(\bar{\theta})$ are computed under 0dB SNR, 500 snapshots, 10 sensors, and $D=11$ sources, as marked by ticks on the $\bar{\theta}$ axis. The mutual coupling model (7) has $c_{1}=0.5 e^{j \pi / 3}, c_{\ell}=c_{1} e^{-j \pi(\ell-1) / 8} / \ell$, and $B=100$.

On the other hand, the maximum element in $\mathbb{X}_{1}^{(2)}$ and the minimum element in $\mathbb{Y}_{1}^{(2)}$ lead to the following difference:

$$
\left(N_{1}+1\right)-\left(1+2 B_{1}\right)-\left(1+2 A_{1}\right)= \begin{cases}1, & \text { if } N_{1} \text { is even } \\ 2, & \text { if } N_{1} \text { is odd }\end{cases}
$$

That is, when $N_{1}$ is even, we will have an additional pair of sensors giving rise to $w(1)$.

Summarizing, super nested arrays are generalizations of nested arrays. First of all, there is a simple closed-form expression for sensor locations, as in the case of nested and coprime arrays (and unlike MRAs). Second, for a fixed number of sensors, the physical aperture and the difference coarray are exactly identical to those of nested arrays, so that the uniform DOF for DOA estimation remains unchanged. In particular, there are no holes in the coarray unlike coprime arrays. Finally, as in coprime arrays, the mutual coupling effects are much less severe than in nested arrays, because the sensors in the dense ULA part of the nested array have now been redistributed. In short, the super nested array combines the best features of nested and coprime arrays.

\section{NUMERICAL RESULTS}

In this section, we compare the DOA estimation performance in the presence of mutual coupling, among nested arrays, coprime arrays, and super nested arrays. The number of sensors is fixed to be 10 . The sensor locations for nested arrays, coprime arrays, and super nested arrays are illustrated in Fig. 1(a), (b), and (c), respectively.

The first row in Fig. 2 lists the associated weight functions. It can be observed that the nested array and the super nested array have the same coarray, ranging from -29 to 29 , and there are no holes. This result is consistent with Theorem 1 and Corollary 1 . On the other hand, the coprime array exhibits holes and the largest contiguous ULA segment in its coarray ranges from -17 to 17 . Hence, the maximum number of resolvable sources for the nested array, the coprime array, and the super nested array are 29,17 , and 29 , respectively. In addition, the weight function $w(1)$ for the nested array, the coprime array, and the super nested array are 5,2 , and 1 , respectively, which is in accordance with (8), (9) and Theorem 2.

Next, the second row of Fig. 2 demonstrates the MUSIC spectrum $P(\bar{\theta})$ for various array configurations. The parameters are 0 dB SNR, 500 snapshots, and $D=11$ uncorrelated sources ( $>$ number of sensors, 10), located at $\bar{\theta}_{i}=-0.2+0.4(i-1) / 10$ for $1 \leq i \leq 11$. The mutual coupling model is based on (7) with $c_{1}=0.5 e^{j \pi / 3}, c_{\ell}=c_{1} e^{-j \pi(\ell-1) / 8} / \ell$, and $B=100$. According to [19], the coarray MUSIC algorithm is evaluated directly from $\mathbf{x}_{\mathbb{S}}$ (6) without using any decoupling algorithms. Note that these results can be further improved by a variety of decoupling algorithms $[5,7-10,12]$. Our setting provides a baseline performance for different arrays. It can be seen from Fig. 2 that only the super nested array is capable of distinguishing 11 sources robustly while both the nested array and the coprime array display false peaks. To compare the result quantitatively, the root-mean-squared error (RMSE) is defined as $E=\left(\sum_{i=1}^{D}\left(\hat{\bar{\theta}}_{i}-\bar{\theta}_{i}\right)^{2} / D\right)^{1 / 2}$, where $\hat{\bar{\theta}}_{i}$ denotes the estimated normalized DOA of the $i$ th source and $\bar{\theta}_{i}$ is the true normalized DOA. The RMSE for the super nested array $(E=0.00282)$ is much smaller that those of the nested and coprime arrays. This result shows that the super nested array is very promising in the presence of mutual coupling, even when no decoupling algorithm is adapted.

\section{CONCLUDING REMARKS}

In this paper, we introduced super nested arrays. We showed that they share many of the good properties of nested arrays but at the same time, have reduced mutual coupling effects. One future direction for further improvement would be to use these arrays in conjunction with techniques which decouple or compensate the effect of mutual coupling [5,7-10,12]. Another direction would be the extension of these ideas to planar arrays. 


\section{REFERENCES}

[1] M. I. Skolnik, Introduction to Radar Systems, McGraw Hill, 3 edition, 2001.

[2] S. Haykin, Array Signal Processing, Prentice-Hall, 1984.

[3] L. Godara, "Application of antenna arrays to mobile communications, Part II: Beam-forming and direction-of-arrival considerations," Proc. IEEE, vol. 85, no. 8, pp. 1195-1245, Aug 1997.

[4] H. L. Van Trees, Optimum Array Processing: Part IV of Detection, Estimation, and Modulation Theory, Wiley Interscience, 2002.

[5] B. Friedlander and A. Weiss, "Direction finding in the presence of mutual coupling," IEEE Trans. Antennas Propag., vol. 39, no. 3, pp. 273-284, Mar 1991.

[6] K. Pasala, "Mutual coupling effects and their reduction in wideband direction of arrival estimation," IEEE Trans. Aerosp. Electron. Syst., vol. 30, no. 4, pp. 1116-1122, Oct 1994.

[7] T. Svantesson, "Mutual coupling compensation using subspace fitting," in Proc. IEEE Sensor Array and Multichannel Signal Processing Workshop, 2000, pp. 494-498.

[8] M. Lin and L. Yang, "Blind calibration and DOA estimation with uniform circular arrays in the presence of mutual coupling," IEEE Antennas Wireless Propag. Lett., vol. 5, no. 1, pp. 315-318, Dec 2006.

[9] F. Sellone and A. Serra, "A novel online mutual coupling compensation algorithm for uniform and linear arrays," IEEE Trans. Signal Process., vol. 55, no. 2, pp. 560-573, Feb 2007.

[10] Z. Ye, J. Dai, X. Xu, and X. Wu, "DOA estimation for uniform linear array with mutual coupling," IEEE Trans. Aerosp. Electron. Syst., vol. 45, no. 1, pp. 280-288, Jan 2009.

[11] J. Dai, D. Zhao, and X. Ji, "A sparse representation method for DOA estimation with unknown mutual coupling," IEEE Antennas Wireless Propag. Lett., vol. 11, pp. 1210-1213, 2012.

[12] E. BouDaher, F. Ahmad, M. G. Amin, and A. Hoorfar, "DOA estimation with co-prime arrays in the presence of mutual coupling," in Proc. European Signal Process. Conf., Nice, France, 2015 .
[13] P. Pal and P. P. Vaidyanathan, "Nested arrays: A novel approach to array processing with enhanced degrees of freedom," IEEE Trans. Signal Process., vol. 58, no. 8, pp. 4167-4181, Aug 2010.

[14] P. P. Vaidyanathan and P. Pal, "Sparse sensing with co-prime samplers and arrays," IEEE Trans. Signal Process., vol. 59, no. 2, pp. 573-586, Feb 2011.

[15] A. T. Moffet, "Minimum-redundancy linear arrays," IEEE Trans. Antennas Propag., vol. 16, no. 2, pp. 172-175, 1968.

[16] H. Taylor and S. W. Golomb, "Rulers, Part I," Tech. Rep. 85-05-01, Univ. Southern Calif., Los Angeles, 1985.

[17] J. Leech, "On the representation of $1,2, \ldots, n$ by differences," J. London Math. Soc., vol. 1, no. 2, pp. 160-169, 1956.

[18] P. Pal and P. P. Vaidyanathan, "Coprime sampling and the MUSIC algorithm," in Proc. IEEE Digit. Signal Process. Signal Process. Educ. Workshop, Jan 2011, pp. 289-294.

[19] C.-L. Liu and P. P. Vaidyanathan, "Remarks on the spatial smoothing step in coarray MUSIC," IEEE Signal Process. Lett., vol. 22, no. 9, pp. 1438-1442, Sept 2015.

[20] Z. Tan, Y. Eldar, and A. Nehorai, "Direction of arrival estimation using co-prime arrays: A super resolution viewpoint," IEEE Trans. Signal Process., vol. 62, no. 21, pp. 5565-5576, Nov 2014.

[21] P. Pal and P. Vaidyanathan, "Correlation-aware techniques for sparse support recovery," in Proc. IEEE Statist. Signal Process. (SSP) Workshop, Aug 2012, pp. 53-56.

[22] S. Qin, Y. Zhang, and M. Amin, "Generalized coprime array configurations for direction-of-arrival estimation," IEEE Trans. Signal Process., vol. 63, no. 6, pp. 1377-1390, March 2015.

[23] H. King, "Mutual impedance of unequal length antennas in echelon," IRE Trans. Antennas Propag., vol. 5, no. 3, pp. 306313, July 1957.

[24] C.-L. Liu and P. P. Vaidyanathan, "Super nested arrays: Linear sparse arrays with reduced mutual coupling - Part I: Fundamentals," IEEE Trans. Signal Process., submitted. 